\title{
Transmembrane Domain 1 of Human Organic Anion Transporting Polypeptide 2B1 Is Essential for Transporter Function and Stability $\mathbf{s}$
}

\author{
Zihui Fang, ${ }^{1}$ Jiujiu Huang, ${ }^{1}$ Jie Chen, Shaopeng Xu, Zhaojian Xiang, and Mei Hong \\ College of Life Sciences (Z.F., J.H., J.C., S.X., Z.X., M.H.) and Guangdong Provincial Key Laboratory of Protein Function and \\ Regulation in Agricultural Organisms (J.H., M.H.), South China Agricultural University, Guangzhou, China
}

Received January 29, 2018; accepted May 24, 2018

\begin{abstract}
Organic anion transporting polypeptides (OATPs, gene symbol $S L C O)$ are important membrane transporter proteins that mediate the uptake of wide ranges of endogenous and exogenous compounds. OATP2B1 has been found in multiple organs and tissues, including the liver, small intestine, kidney, brain, placenta, heart, skin, as well as skeletal muscle, and is proposed to be involved in the uptake of orally administered drugs. Quite a few reports have demonstrated that transmembrane domains (TMs) are crucial for proper functions of OATP family members. Comparative modeling proposed that TM1, along with TM2, 4, and 5 of the $\mathrm{N}$-terminal half of OATP2B1, may be localized within the substrate interaction pocket and are important for uptake function of the transporter. Alanine scanning of the putative transmembrane domain 1 of
\end{abstract}

\section{Introduction}

Organic anion transporting polypeptides (OATPs, gene symbol $S L C O$ ) belong to the solute carrier family and mediate sodium-independent transport of various endogenous and exogenous compounds (Hagenbuch and Gui, 2008). Bile salts, hormones and their conjugates, toxins, and a wide range of drugs have been found to be transported by OATPs. Twelve human OATP family members have been found so far: OATP1A2, 1B1, 1B3, 1B7, 1C1, 2A1, 2B1, 3A1, 4A1, 4C1, 5A1, and 6A1 (Hagenbuch and Meier, 2003; Nakanishi and Tamai, 2012). However, SLCO1B7 was proposed as a pseudogene because OATP1B7 is considered as nonfunctional (Stieger and Hagenbuch, 2014). Some OATP family members are predominantly expressed in certain organs or tissues. For example, OATP1B1 and OATP1B3 are found only in the liver, whereas others were reported as being expressed ubiquitously (König et al., 2006). Due to their broad substrate specificity, wide tissue distribution, and the involvement of drug-drug

This work was supported by the National Natural Science Foundation of China [Grant 81373473] and National Natural Science Foundation of Guangdong Province [Grant 2015A030312005] to M.H.

${ }^{1}$ Z.F. and J.H. contributed equally to this work.

https://doi.org/10.1124/mol.118.111914.

S This article has supplemental material available at molpharm. aspetjournals.org.
OATP2B1 revealed that substitution of L58 with alanine dramatically altered the $\mathrm{K}_{\mathrm{m}}$ value, and mutation of V52, H55, Q59, and L69 resulted in significantly reduced substrate turnover number, whereas A61V, Q62A, and S66A exhibited significant change in both $\mathrm{K}_{\mathrm{m}}$ and $\mathrm{V}_{\max }$ values. In addition, phenylalanine at position 51 seems to play an important role in maintaining proper folding of OATP2B1 because alanine replacement of F51 caused accelerated degradation of the transporter protein. Although proteasome and lysosome inhibitors could partially recover protein level, the mutant transporter remained nonfunctional. Taken together, the identification of nine essential amino acid residues within TM1 of OATP2B1 suggested that the transmembrane domain is important for maintaining proper function of the transporter.

ABBREVIATIONS: ES, estrone-3-sulfate; MG132, carbobenzoxy-Leu-Leu-leucinal; NHS-SS-biotin, sulfosuccinimidyl 2-(biotinamido)-ethyl-1,3dithiopropionate; OATP, organic anion transporting polypeptide; TM, transmembrane domain.

interactions, OATPs have been extensively recognized as key determinants for drug absorption, distribution, and excretion (Shitara et al., 2005; Poirier et al., 2007).

OATP2B1 has been found in multiple organs and tissues, including the liver, small intestine, kidney, brain, placenta, heart, skin, as well as skeletal muscle (Tamai et al., 2000; Kullak-Ublick et al., 2001; Hagenbuch and Meier, 2004; Niessen et al., 2009; Knauer et al., 2010, 2013). In the liver, OATP2B1 is involved in hepatic uptake of a wide spectrum of xenobiotics, and many organic anions, mono- and dicarboxylic acids (Kobayashi et al., 2003; Sai et al., 2006), steroid hormones and their derivatives (Grube et al., 2006), as well as drugs such as rifamycin SV (Vavricka et al., 2002), pravastatin (Kobayashi et al., 2003), cyclosporine, and gemfibrozil (Tamai et al., 1997) are inhibitors of the transporter. OATP2B1 exhibits high expression in the apical membrane of human intestinal epithelial cells and may function as a pH-dependent organic anion transporter (Kis et al., 2010). Since the physiologic microclimate $\mathrm{pH}$ in the intestinal lumen is weakly acidic, OATP2B1 that shows higher activity at acidic $\mathrm{pH}$ is believed to be involved in the uptake of drugs administered orally (Kobayashi et al., 2003; Tamai, 2012). Although extensive studies have been carried out to identify substrates of OATPs, the underlying mechanisms of substrate binding 
and/or recognition remain largely unclear because highresolution crystal structures of mammalian drug transporters are still not available (Niessen et al., 2009).

Transmembrane domains (TMs) have been demonstrated to be important for proper functions of various transporters. Previous studies have identified quite a few essential amino acids located within transmembrane domains of OATP members. For example, amino acid residues within TM2 (Li et al., 2012), TM6 (Huang et al., 2013), TM10 (Gui and Hagenbuch, 2009; Ohnishi et al., 2014), and TM11 (Weaver and Hagenbuch, 2010; Hong et al., 2015) were shown to be crucial for proper function of OATP1B1, and TM8 (Miyagawa et al., 2009) is believed to be important for substrate recognition of the transporter. As for OATP1B3, K41 in TM1 and R580 in TM11 are believed to be pivotal to the transporter function (Glaeser et al., 2010). In addition, G45 within TM1 along with Y537, S545, and T550 within TM10 (Gui and Hagenbuch, 2008; DeGorter et al., 2012) were demonstrated to be critical for the transport of OATP1B3-specific substrate CCK8. TM6 of OATP1A2 seems to be an important region for substrate binding, protein stability, and trafficking (Chan et al., 2015). However, little information is available for the role of TMs within OATP2B1.

Comparative modeling proposed that transmembrane helix 1 along with TM2, 3 , and 5 of the N-terminal half of OATP2B1 may be involved in formation of the substrate interaction pore (Meier-Abt et al., 2005). In the present study, alanine scanning of the putative TM1 of OATP2B1 identified nine amino acid residues that are essential for the transporter function. Kinetic analysis of the functionally impaired mutants showed that $\mathrm{K}_{\mathrm{m}}$ and/or $\mathrm{V}_{\max }$ values of most of the mutants were affected, and that F51A exhibited dramatically reduced protein expression. The identification of nine critical amino acid residues within TM1 of OATP2B1 suggested that the transmembrane domain is important for maintaining proper function of the transporter protein.

\section{Materials and Methods}

Materials. $\left[{ }^{3} \mathrm{H}\right]$ Estrone-3-sulfate (ES) and $\left[{ }^{3} \mathrm{H}\right]$ taurocholic acid were obtained from PerkinElmer Life Sciences (Waltham, MA). Sulfosuccinimidyl 2-(biotinamido)-ethyl-1,3-dithiopropionate (NHSSS-biotin) and streptavidin-agarose beads were purchased from Thermo Scientific (Rockford, IL). All other reagents were from Sigma-Aldrich (St. Louis, MO) except when otherwise stated.

Site-Directed Mutagenesis. Mutants were generated using a QuikChange Lightning Site-Directed Mutagenesis Kit (Agilent, Santa Clara, CA). The pReceiver M07 vector that contains the SLCO2B1 cDNA and 3-hemagglutinin tags at the $\mathrm{C}$ terminus was obtained from GeneCopoeia (Rockville, MD) and used as a template for the mutagenesis. All mutant sequences were confirmed by full-length sequencing (Thermo Scientific).

Cell Culture and Transfection of Plasmid Constructs into Cells. HEK293 cells were grown in Dulbecco's modified Eagle's medium (Thermo Scientific) supplemented with $10 \%$ fetal bovine serum at $37^{\circ} \mathrm{C}$ and $5 \% \mathrm{CO}_{2}$. Confluent cells in a 48 -well or six-well plate were transfected with DNA plasmid using Lipofectamine 2000 reagent (Thermo Scientific) following the manufacturer's instructions and used for transport assay and cell surface biotinylation 48 hours after transfection.

Cell Surface Biotinylation and Western Blotting. Cell surface expression level of OATP2B1 and mutants was examined using the membrane-impermeable biotinylation reagent NHS-SS-biotin as described before (Li et al., 2012; Huang et al., 2013). In brief, HEK293 cells expressing OATP2B1 or mutants were labeled on ice with NHSSS-biotin in two successive 20-minute incubations and lysed with radioimmunoprecipitation assay buffer $[50 \mathrm{mM}$ Tris, $150 \mathrm{mM} \mathrm{NaCl}$, $0.1 \%$ SDS, $1 \% \mathrm{NP} 40$, and protease inhibitors phenylmethylsulfonyl fluoride $(200 \mu \mathrm{g} / \mathrm{ml})$ and leupeptin $(3 \mu \mathrm{g} / \mathrm{ml}), \mathrm{pH} 7.4]$. The labeled proteins in supernatant were then pull downed by streptavidinagarose beads, released in $4 \times$ Laemmli buffer and loaded onto a 7.5\% SDS-polyacrylamide electrophoresis gel, transferred electrophoretically to a polyvinylidene difluoride membrane (Millipore, Billerica, MA), and detected with anti-hemagglutinin antibody (Cell Signaling Technology, Danvers, MA).

Uptake Assay. HEK293 cells in a 48-well plate were used for transport measurement as described before ( $\mathrm{Li}$ et al., 2012; Huang et al., 2013) with minor modification. In brief, cells were incubated with uptake solution that contained $\left[{ }^{3} \mathrm{H}\right] \mathrm{ES}(\mathrm{pH} 7.4)$ or $\left[{ }^{3} \mathrm{H}\right]$ taurocholic acid ( $\mathrm{pH} 5.0$ ) at $37^{\circ} \mathrm{C}$ for 2 minutes ( 1 minute for kinetic analysis), and the reaction was stopped by ice-cold phosphate-buffered saline solution. Cells were then washed twice with cold phosphate-buffered solution, solubilized in $0.2 \mathrm{~N} \mathrm{NaOH}$ followed by neutralization with $0.2 \mathrm{~N} \mathrm{HCl}$, and radioactivity of the cell lysate was measured using a Triathler-Hidex (Hidex, Turku, Finland) liquid scintillation counter. The uptake count was standardized by the amount of protein in each well.

Statistical Analysis. Data statistical analysis was carried out using one-way analysis of variance with Bonferroni's post hoc test. Differences between means are regarded as significant if $P<0.05$.

\section{Results}

Characterization of OATP2B1 Function in HEK293 Cells. To see whether OATP2B1 could be properly expressed and function in HEK293 cells, we first analyzed cell surface expression and kinetic parameters of OATP2B1. As shown in Fig. 1, OATP2B1 was expressed and correctly targeted to the plasma membrane and exhibited a $\mathrm{K}_{\mathrm{m}}$ value of $6.76 \pm 0.71$ $\mu \mathrm{M}$, which is consistent with the previously reported $\mathrm{K}_{\mathrm{m}}$ of $8.09 \pm 1.67 \mu \mathrm{M}$ (Nozawa et al., 2004) for OATP2B1 expressed in HEK293 cells. These results indicated that OATP2B1 was properly expressed and functioned within HEK293 cells.

Effect of TM1 Mutants on OATP2B1 Uptake Function and Protein Expression. To analyze critical amino acid residues within TM1, we performed alanine scanning. Each of the amino acid residues located within putative transmembrane domain 1 of OATP2B1 (Fig. 2) was mutated to alanine, and uptake function of each mutant was measured. Interestingly, most of the alanine mutants showed statistically significant change of ES uptake, and more than half of the mutants ( 11 out of 21 ) exhibited greater than $50 \%$ reduction of the transport function (Fig. 3), indicating that TM1 is important for transport activity of OATP2B1. Since OATP2B1 is a membrane protein, and proper targeting of the transporter to plasma membrane is essential for its function, cell surface expression of the mutants with more than 50\% decreased function was investigated. As shown in Fig. 4A, with the exception of F51A, all mutants exhibited cell surface protein levels comparable to wild-type OATP2B1. Total protein expression was also analyzed for the mutants, and a similar trend was observed (Fig. 4B).

Kinetic Analysis of TM1 Mutants with Significantly Reduced Estrone-3-Sulfate Uptake Function. To evaluate whether the effect on uptake function of these mutants was due to reduced protein level on the plasma membrane, ES uptake of different mutants was normalized with their cell surface protein expression (Fig. 4C). It was shown that after 
A

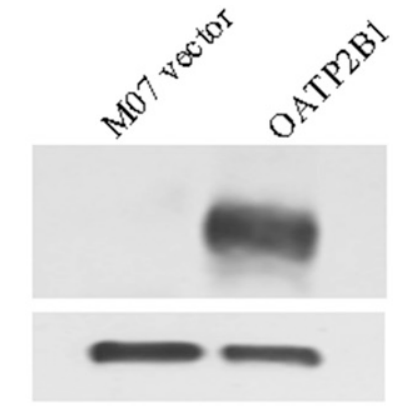

Integrin

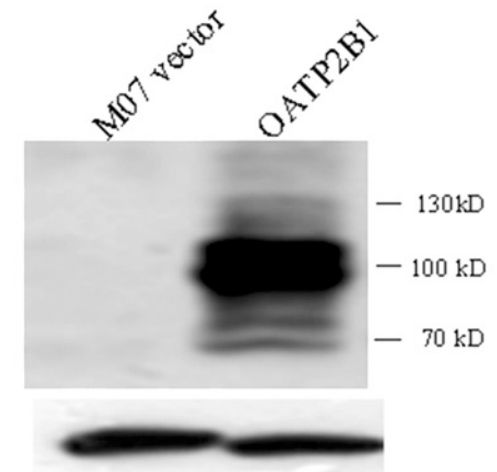

Actin
B

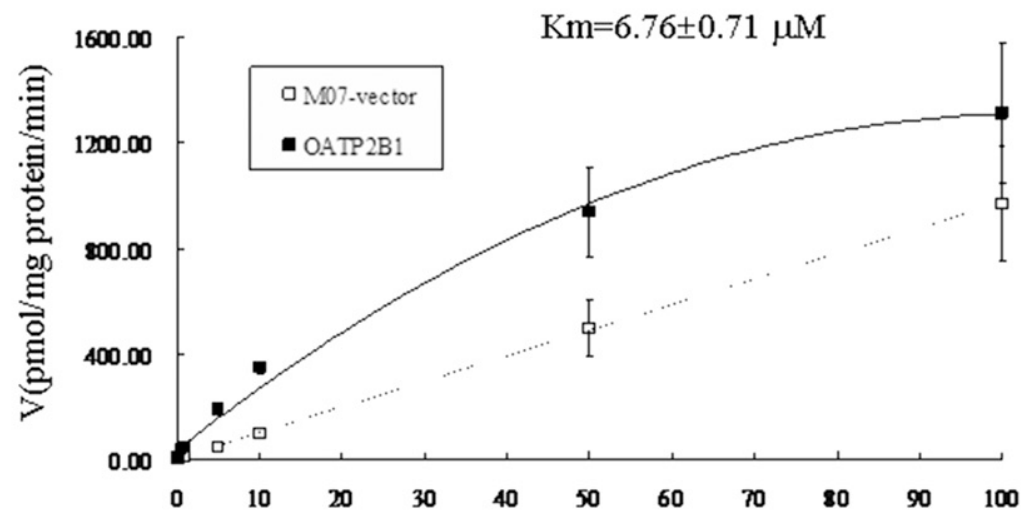

ES concentrations $(\mu \mathrm{M})$

Fig. 1. Characterization of OATP2B1 in HEK293 cells. (A) Cell surface (upper panel) and total protein (lower panel) expression of OATP2B1 in HEK293 cells. Cells were biotinylated and lysed with radioimmunoprecipitation assay buffer, and the biotin-labeled cell surface proteins were then precipitated with streptavidin-agarose beads, separated by SDS-PAGE, followed by western blotting with anti-hemagglutinin antibody. Or cells were lysed with radioimmunoprecipitation assay buffer and subjected to analysis as described earlier. The same blot was probed with integrin or actin as protein loading control. (B) Kinetic analysis of OATP2B1. Uptake ( $\mathrm{pH} 7.4$ ) of ES was measured at concentrations ranging from 0.05 to $100 \mu \mathrm{M}$ at $37^{\circ} \mathrm{C}$ at a 1 -minute interval. The results represent data from three experiments, with duplicate measurements for each sample. The results shown are means \pm S.D. $(n=3)$. $\mathrm{K}_{\mathrm{m}}$ (shown as mean \pm S.D.) was determined with nonlinear regression of the Michaelis-Menten equation incorporated in GraphPad Prism 5 (GraphPad Software, La Jolla, CA).

protein level adjustment, ES uptake of K49A, F51A, and I65A was partially recovered to more than $50 \%$ of that of wild-type OATP2B1, suggesting that these residues may have affected protein expression of the transporter, which in turn led to reduced uptake function. On the other hand, the other eight mutants, i.e., V52A, H55A, L58A, Q59A, A61V, Q62A, S66A, and L69A, still exhibited more than 50\% decrease of transport activity, indicating that replacement of these residues may change the interaction of the transporter with substrate. Therefore, kinetic analysis of ES uptake was performed, and as shown in Table 1, A61V, Q62A, and S66A affected binding affinity as well as substrate turnover number of the transporter; whereas L58A only showed increased $\mathrm{K}_{\mathrm{m}}$ value, V52A, H55A, Q59A, and L69A only showed altered $\mathrm{V}_{\max }$.

To see whether only ES uptake was affected by mutation at these positions, we analyzed transport activity of taurocholate by OATP2B1 and the functionally impaired mutants as well. As shown in Fig. 5, mutants that showed more than 50\% decrease in ES uptake also exhibited significant reduction $(>50 \%)$ of transport activity for taurocholate, suggesting these residues are involved in uptake of both substrates.

Alanine Replacement of F51 Affects Protein Stability of OATP2B1. To see whether the significantly reduced expression of F51A was due to an increased degradation of
OATP2B1 ----MGPRIGPAGEVPQVPDKETKATMGTENTPGGKASPDPQDVRPSVFHNIKLFVLCHSLLQLAQ-LIISGYLK

OATP $1 B 1$--_-_-_-_-_-_-_-_-_

OATP $1 B 3$-------'MDQHQHLNKTAESASSEKKKTRRCNGFKMFLAALSFSYIAK-ALGGIIMK

OATP 1C1 ------MDTSSKENI QLFCKTSVQPVGRPSFKTEYPSSEEKQPCCGELKVFLCALSFVYFAK-ALAEGYLK

OATP $1 \mathrm{~A} 2$ -MGETEKRIETHRI RCLSKLKMFLLAITCAFVSK-TLSGSYMN

OATP2A1 --------MGLLPKLG-----ASQGSDTSTSRAG-----RCARSVFGNIKVFVLCQGLLQLCQ-LLYSAYFK

OATP4C1 PQEPQKSPEPSLPSAPPNVSEEKLRSLSLSEFEEGSYGWRNFHPCLQRCNTPGGLLHYCLLAVTQGIVVNGLVN

OATP6A1 LPEALIRFG-GFRKRKKAKSSVSKKPGEVDDSLEQPCGLGCLVSTCCECCNNIRGFIFYCILLICQGWVG-LID

OATP4A1 PLDTSKQPLCQLWAEKHGARG-THEVRYVSAGQSVACGWWAFAPPCLQVLNTPKGILFFLCAAAFLQGMTVNGF IN

OATP3A1 -------MQGKKPGGSSGGGRSGELQGDEAQRNKKKKKKVSCFSNIKIFLVSECALMLAQ-GTVGAYLV

OATP5A1 GPNPLAPSPSAPSTSAGLGDCNHRVDLSKTFSVSSALAMLQERRCLYWLTDSRCFLVCMCFLTFIQALUVSGYLS
Fig. 2. Sequence comparison of OATP family members. Multiple sequence alignment of different OATPs was performed with Clustal W (www.clustal.org). The putative transmembrane domain 1 of OATP family members was predicted according to the Kyte-Doolittle hydrophobicity scale. Only partial sequences are shown. The corresponding sequences of TM1 are in bold. 


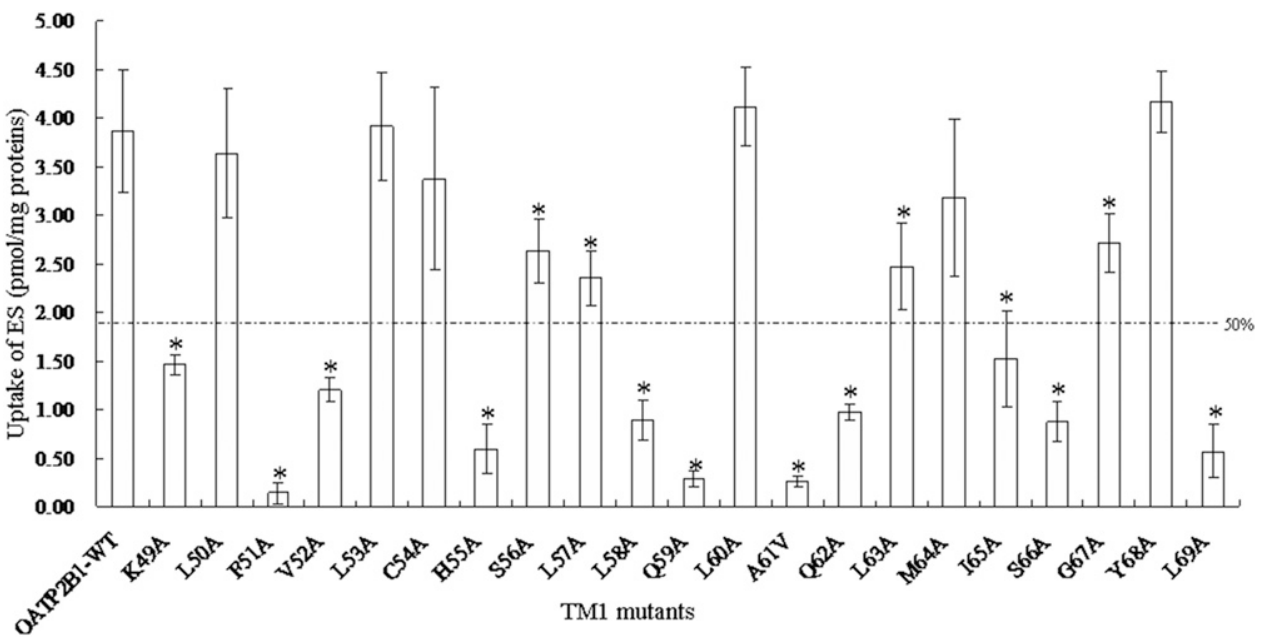

Fig. 3. Uptake of estrone-3-sulfate by OATP2B1 transmembrane domain $1 \mathrm{mu}-$ tants. Uptake of $50 \mathrm{nM} \mathrm{ES}$ ( $\mathrm{pH} 7.4$ ) by cells expressing OATP2B1 and its alanine-substituted mutants was measured at $37^{\circ} \mathrm{C}$ at a 2 -minute interval. Net uptake was obtained by subtracting the uptake of cells transfected with empty vector from cells expressing wild-type OATP2B1 (OATP2B1-WT) or mutants. The results represent data from three experiments, with duplicate measurements for each sample. The results shown are means \pm S.D. $(n=3)$. Asterisks indicate significant difference compared with wild-type OATP2B1 $(P<0.05)$.

the transporter, the mutant was treated with proteasome inhibitor MG132 (carbobenzoxy-Leu-Leu-leucinal) or lysosome inhibitor bafilomycin A1. As shown in Fig. 6A, MG132 treatment resulted in partial recovery of the total protein (left panel). In addition, it was observed that only the immature form $(\sim 72 \mathrm{kD})$ of the transporter was recovered after MG132 treatment; the mature form $(\sim 100 \mathrm{kD})$ of the protein remained almost undetectable. Consistent with this result, cell surface

A
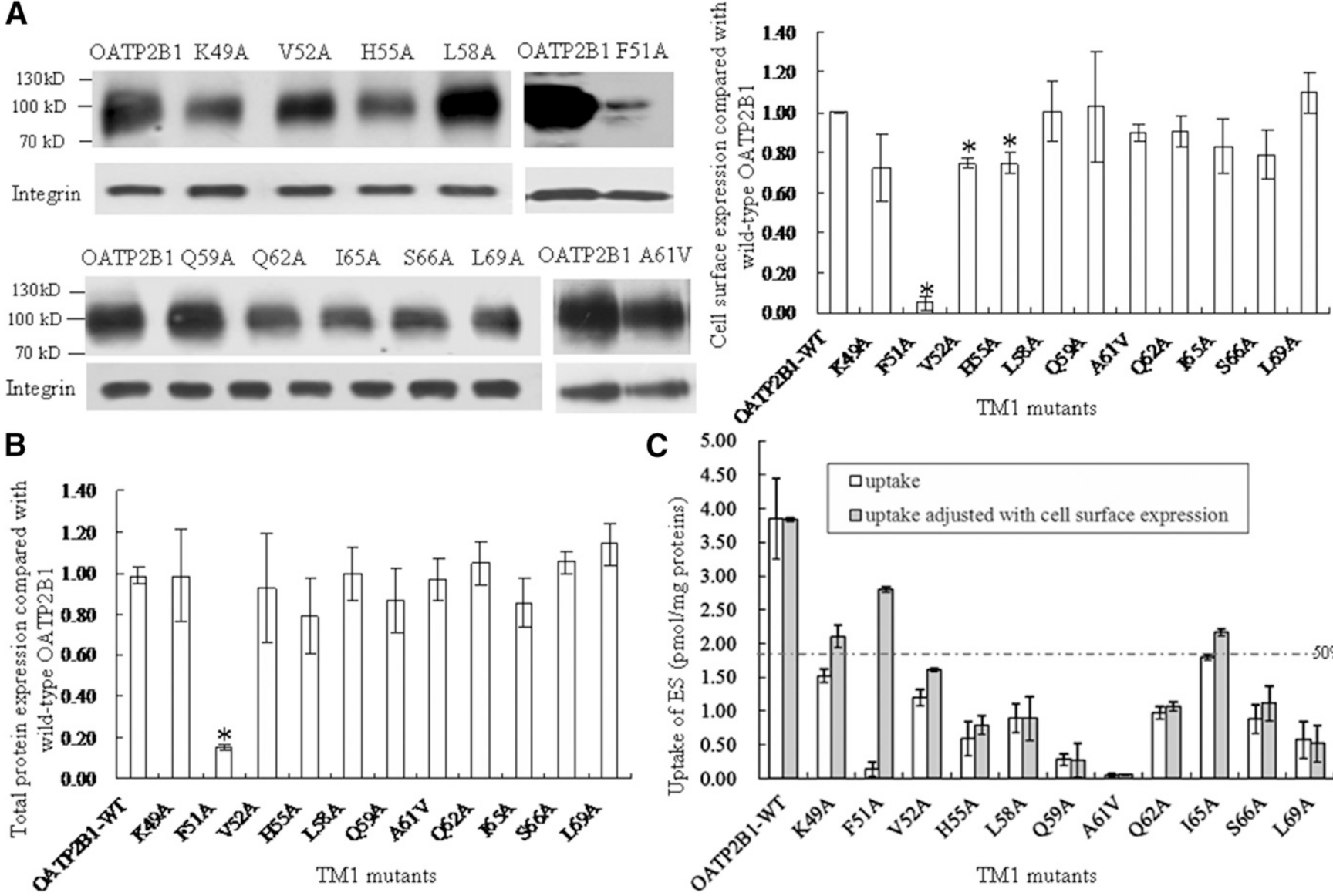

TM1 mutants

Fig. 4. Protein expression of TM1 mutants with significantly reduced function. (A) Cell surface expression of OATP2B1 and TM1 mutants. (B) Total protein expression of OATP2B1 and mutants. For cell surface expression of OATP2B1 and mutants, cells were biotinylated, and the biotin-labeled cell surface proteins were precipitated with streptavidin-agarose beads, separated by SDS-PAGE, followed by western blotting with anti-HA antibody. The same blot was probed with integrin antibody as surface protein loading control. A representative blot was shown for cell surface protein expression of OATP2B1 and mutants. Intensity of protein bands was quantified with ImageJ (National Institutes of Health, Bethesda, MD) and compared relative to the wild type. The results shown are means \pm S.D. $(n=3)$. Asterisks indicate significant difference compared with wild-type OATP2B1 (OATP2B1-WT) $(P<0.05)$. (C) Estrone-3-sulfate uptake of different mutants was normalized with their corresponding protein expression levels. The results represent data from three experiments. The results shown are means \pm S.D. $(n=3)$. 
TABLE 1

Kinetic parameters of estrone-3-sulfate transport by wild-type OATP2B1 and TM1 mutants

Uptake of ES was measured at concentrations ranging from 0.05 to $50 \mu \mathrm{M}$ for wildtype OATP2B1 and mutants at $37^{\circ} \mathrm{C}$ at a 1-minute interval and normalized with cell surface expression of the transporter. Transporter kinetic parameters were determined with nonlinear regression of Michaelis-Menten equation incorporated in GraphPad Prism 5. The results shown are means \pm S.D. $(n=3)$.

\begin{tabular}{lcc}
\hline & $\mathrm{K}_{\mathrm{m}}$ & $\frac{\mathrm{V}_{\max }}{n}$ \\
\cline { 2 - 3 } & $\mu M$ & $\begin{array}{c}\mathrm{pmol} / \mathrm{mg} \\
\mathrm{protein} / \mathrm{min}\end{array}$ \\
\hline OATP2B1 & $6.40 \pm 0.30$ & $351 \pm 1$ \\
F51Y & $7.30 \pm 1.70$ & $365 \pm 6$ \\
V52A & $5.28 \pm 1.54$ & $71.4 \pm 3.4^{*}$ \\
H55A & $6.75 \pm 1.17$ & $86.5 \pm 2.0^{*}$ \\
L58A & $18.4 \pm 2.6^{*}$ & $314 \pm 29$ \\
Q59A & $6.27 \pm 0.65$ & $27.9 \pm 3.1^{*}$ \\
A61V & $24.9 \pm 4.2^{*}$ & $213 \pm 20^{*}$ \\
Q62A & $7.36 \pm 1.01^{*}$ & $134 \pm 1^{*}$ \\
S66A & $10.1 \pm 0.7^{*}$ & $167 \pm 4^{*}$ \\
L69A & $7.20 \pm 1.30$ & $73.3 \pm 4.8^{*}$ \\
\hline
\end{tabular}

*Values significantly different $(P<0.05)$ from that of wild-type OATP2B1.

expression of F51A remained negligible even after MG132 treatment (right panel). Accordingly, MG132 treatment showed a marginal effect on uptake function of F51A (Fig. $6 \mathrm{~B})$. Treatment with the vacuolar proton ATPase inhibitor bafilomycin A1 resulted in a significantly elevated level of F51A. Further analysis revealed that the lysosome inhibitor also increased cell surface expression of F51A (Fig. 6C). Similar result was observed on treatment with additional lysosomal inhibitors such as ammonia chloride and leupeptin (data not shown). However, uptake function of the mutant transporter was not affected by bafilomycin A1 treatment (Fig. 6D).

Conservative Replacement of Amino Acids Important for OATP2B1 Function. To investigate whether the side chain structures of these critical amino acids are essential for OATP2B1 function, we substituted the residues with conservative amino acids. As shown in Fig. 7, replacement of hydrophobic residues (V52, L58, and L69) with an amino acid that contains similar side chain structure partially recovered transport function of the protein. In addition, substitution of F51 with tyrosine increased both protein expression and transport activity (Fig. 7). On the other hand, conservative replacement of $\mathrm{H} 55$ with lysine significantly reduced uptake function and protein expression of the transporter, whereas substitution of Q62 with asparagine exhibited decreased transport activity but showed similar cell surface expression compared with Q62A. No significant change of protein expression was observed for other mutants (data not shown). Since F51Y partially recovered uptake function of ES, we performed kinetic analysis of the mutant. As shown in Table 1, after normalization with cell surface expression, $\mathrm{K}_{\mathrm{m}}$ and $\mathrm{V}_{\max }$ of F51Y were comparable to those of wild-type OATP2B1, indicating that phenylalanine at this position may not directly interact with the substrate (Table 1).

\section{Discussion}

Although quite a few reports have shown that OATP2B1 is involved in the transport of a wide range of compounds, information related to the structure-function relationship of OATP2B1 is still limited. OATP2B1 has been demonstrated to contain multiple substrate binding sites in Xenopus oocytes (Shirasaka et al., 2012) and Caco-2 cells (Kis et al., 2010). However, in our current study, the transporter only showed monophasic transport of estrone-3-sulfate. The difference may be attributable to the different systems used. In an earlier study of OATP2B1 expressed in HEK293 cells, it was shown that the transporter exhibited single saturation kinetics as well (Nozawa et al., 2004).

Transmembrane domains have been demonstrated to play important roles in proper function of OATP1B1, 1B3, and 1A2. In the present study, we performed alanine scanning of putative TM1 of OATP2B1 and found that alanine substitution of 11 residues within the transmembrane domain resulted in more than $50 \%$ reduction of ES uptake by the transporter. After normalization with cell surface protein level, there were still eight mutants exhibiting greatly reduced transport function ( $>50 \%$, Fig. $4 \mathrm{C}$ ). When locations of these essential residues were analyzed, it was found that from V52 on, alanine replacement of every three and/or four residues, i.e., V52, H55, L58, Q59, A61, Q62, S66, L69, resulted in a mutant that showed significantly decreased transport function. Since amino acid residues spaced three or four residues apart in protein primary sequence are spatially close to each other in an $\alpha$-helix (Tymoczko et al., 2015), these TM1 residues may be lined up at the same side of the $\alpha$-helix, interacting with OATP2B1 substrates. A similar phenomenon was observed in our previous study of OATP1B1. It was found that several amino acid residues along TM11 are crucial for uptake function of the transporter, and they are located three and/or four residues apart from each other (Hong et al., 2015).

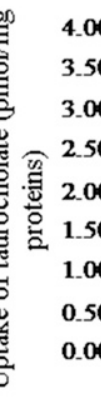

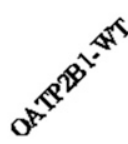

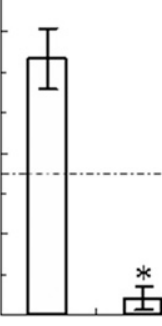

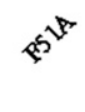

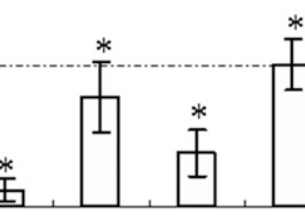
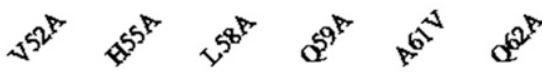

TM1 mutants
Fig. 5. Taurocholate uptake of mutants with significantly reduced ES transport function. Uptake of $1 \mu \mathrm{M}$ taurocholate (pH 5.0) by HEK293 cells expressing OATP2B1 and mutants was measured at $37^{\circ} \mathrm{C}$ at a 2-minute interval. Net uptake was obtained by subtracting the uptake by cells transfected with empty vector from cells expressing wild-type OATP2B1 (OATP2B1-WT) or mutants. The results represent data from three experiments, with duplicate measurements for each sample. The results shown are means \pm S.D. $(n=3)$. Asterisks indicate significant difference compared with wild-type OATP2B1 $(P<0.05)$. 
A

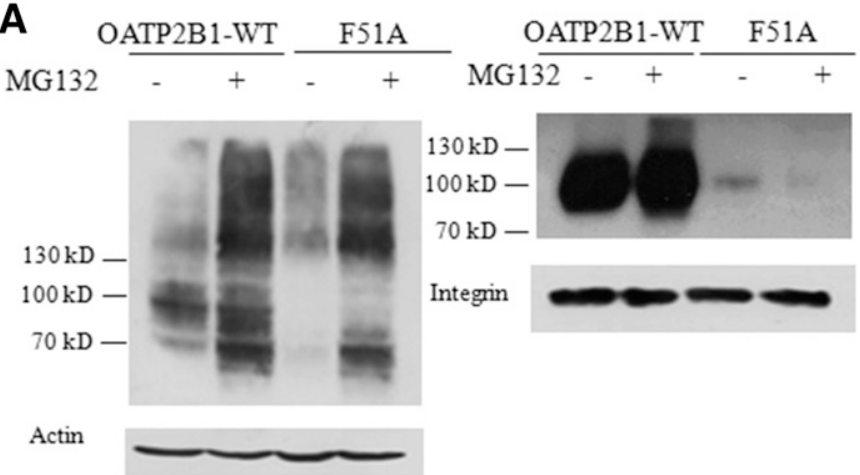

C

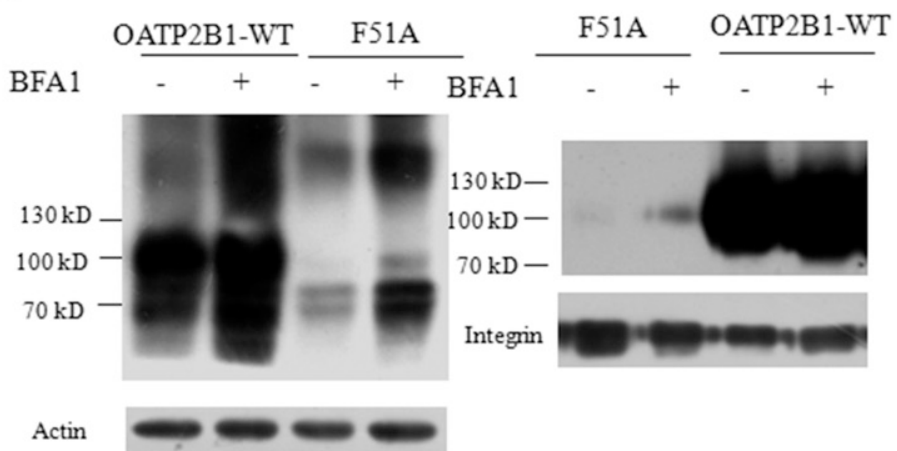

B

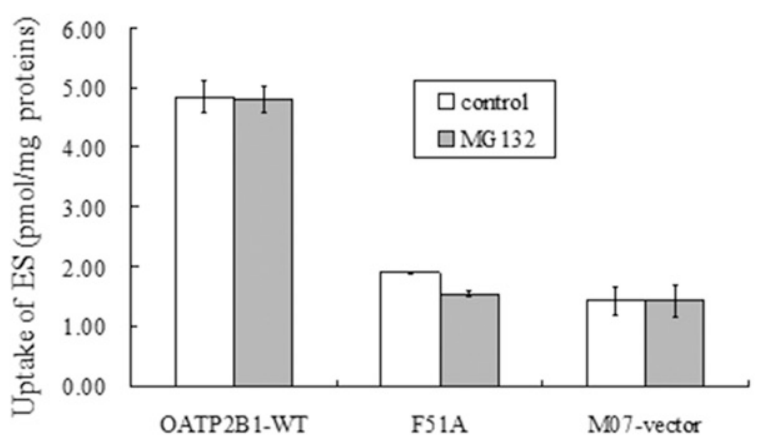

D

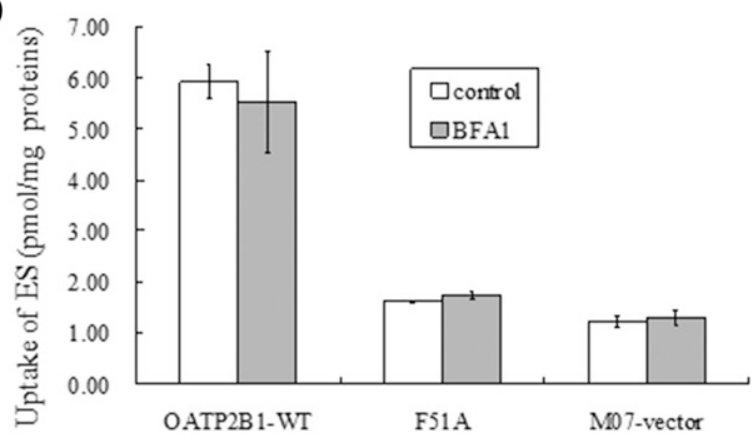

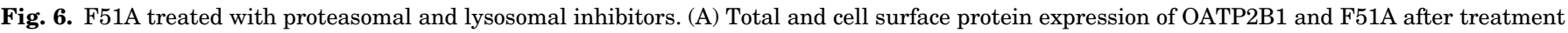

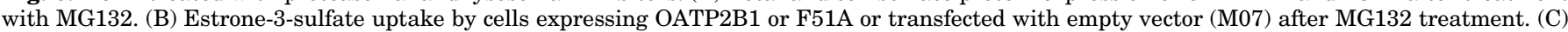

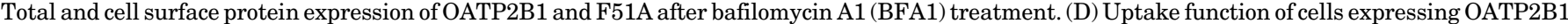

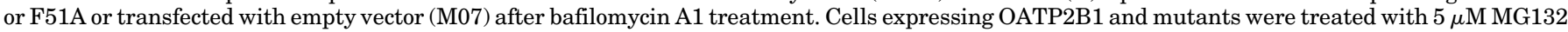

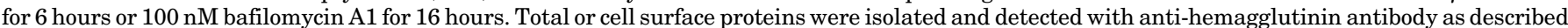

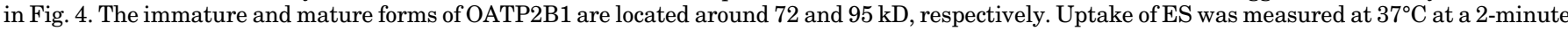

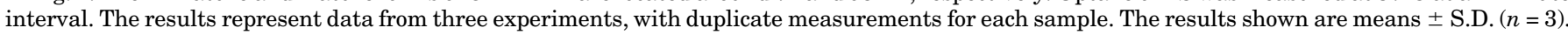

Indeed, homology modeling (Biasini et al., 2014) of OATP2B1 (Supplemental Material) with Escherichia coli glycerol-3phosphate transporter (Protein Data Bank: 1pw4, Supplemental Material) as template revealed that V52, H55, L58, Q59, A61, Q62, S66, and L69 are all localized along one side the $\alpha$-helix, facing the inner channel formed among different transmembrane domains (Fig. 8). These results are consistent with previous reports of putative computer models of OATP family members. It was proposed that for OATP1B3, the $\mathrm{N}$-terminal half of TMs 1, 2, 4, and 5 and the C-terminal half of TMs 7, 8, 10, and 11 likely face the central pore, and that the presence of a central pore may be a conserved feature and of functional significance for OATP members (Meier-Abt et al., 2005). A more recent structural model of OATP2B1 also revealed that amino acid residues of TMs $1,2,4,5,7,8,10$, and 11 may be involved in the formation of the putative substrate translocation pathway of the transporter (Bian et al., 2016). It should be noted that the sequence identity of glycerol-3-phosphate transporter compared with OATP2B1 is relatively low (12.87\%). However, homology modeling using the transporter covers the most extended area, i.e., from TM1 to TM12 of OATP2B1, among different templates with comparable identity. A similar structure was obtained using the human glucose transporter family member 1 (Protein Data Bank: 4pyp), which has the highest sequence identity (15.82\%) compared with OATP2B1 but exhibits a lower coverage along the sequence (data not shown) as template.
Among the identified residues, V52 is highly conserved, with 10 OATPs having a nonpolar, hydrophobic amino acid at this position (Fig. 2). At position L58, other OATPs except the two OATP1B family members have the same residue or a residue with similar structure. Similarly, the residues at position 69 are nonpolar and hydrophobic in OATP2B1 and other OATPs, with the exception of OATP1B1, 1B3, and 1A2. The conservative replacement of these residues partially recovered uptake function of the transporter, suggesting the hydrophobic property of these residues may be important for transport function of OATP members. A61 seems to be only conserved among OATP2B1 and most of the OATP1 subfamily members, whereas a polar residue is found in other OATPs. At position Q62, the residue is conserved in other OATPs except those of the OATP1 family, which all contain a positively charged lysine residue at this position. On the other hand, H55, Q59, and S66 seem to be unique for OATP2B1 and may be involved in OATP2B1-specific functions.

The protein expression of F51A was dramatically reduced, suggesting phenylalanine at this position may play an important role in protein stability. Proteasome inhibitor MG132 partially recovered the immature form of the transporter $(\sim 72$ $\mathrm{kD}$ ), whereas lysosomal inhibitor bafilomycin A1 increased both the immature and mature forms $(\sim 100 \mathrm{kD})$ of F51A, and the cell surface expression of the transporter was partially recovered. However, the recovered F51A is not functional. Both the proteasome and lysosome are important sites for 
A
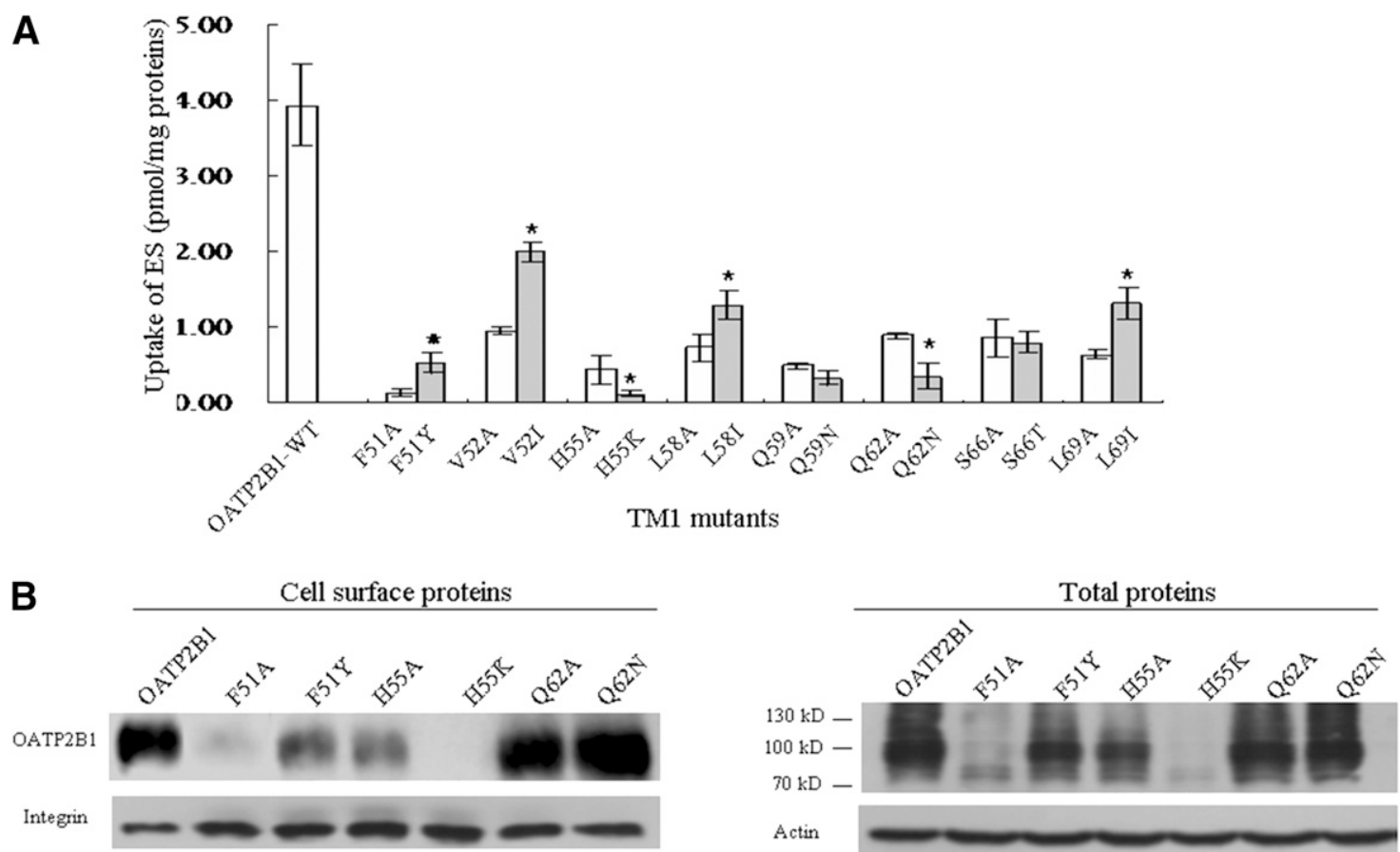

Fig. 7. Uptake function and protein expression of additional mutants with conservative replacement. (A) Uptake of nonconservative and conservative mutants. Uptake of ES by cells expressing OATP2B1 and its mutants was measured at $37^{\circ} \mathrm{C}$ at a 2 -minute interval. Net uptake was obtained by subtracting the uptake of cells transfected with empty vector from cells expressing wild-type OATP2B1 (OATP2B1-WT) or mutants. The results represent data from three experiments, with duplicate measurements for each sample. The results shown are means \pm S.D. $(n=3)$. Asterisks indicate significant difference between mutants of the same amino acid residue $(P<0.05)$. (B) Protein expression of mutants showing significant change in uptake function. Cell surface (left panel) or total proteins (right panel) of OATP2B1 and different mutants were extracted and analyzed as described in Fig. 4.

the degradation of misfolded proteins (Ciechanover, 2005). These data suggested that alanine replacement of F51 may result in a protein that is recognized by the quality control machinery as incorrectly folded and hence targeted for degradation by the proteasome and lysosome. However, mutation of F51 does not seem to affect maturation of the protein because blocking the lysosomal pathway could partially recover the mature form of the transporter. Conservative replacement with tyrosine resulted in partial recovery of transport function as well as cell surface protein expression, which indicated that the aromatic group at F51 is important for proper protein folding.

In the case of H55 and Q62, conservative replacement even reduced uptake function of the mutants, suggesting side chain structures of these residues are irreplaceable for the uptake function. Alanine replacement of L58, Q62, and S66 and valine substitution of A61 significantly decreased the binding affinity for ES, indicating that these residues may be involved in substrate binding. All mutants except L58A showed significantly reduced $\mathrm{V}_{\max }$, which suggested that these amino acid residues are crucial for substrates turnover by OATP2B1 as well. These results indicated that L58, A61, Q62, and S66 may be part of the substrate binding site and/or translocation pathway.

In the present study, nine critical amino acids were identified within transmembrane domain 1 of OATP2B1. Most of these residues are important for substrate interaction, whereas F51 is crucial for correct folding of the transporter protein. Although so far there has not been any report relating biologic significance to nonsynonymous genetic polymorphisms in OATP2B1 TMs, our search in the National Center for Biotechnology Information database found reports of missense residues within different TMs of the transporter; in particular, a V52I mutant with a minor allele frequency of
A

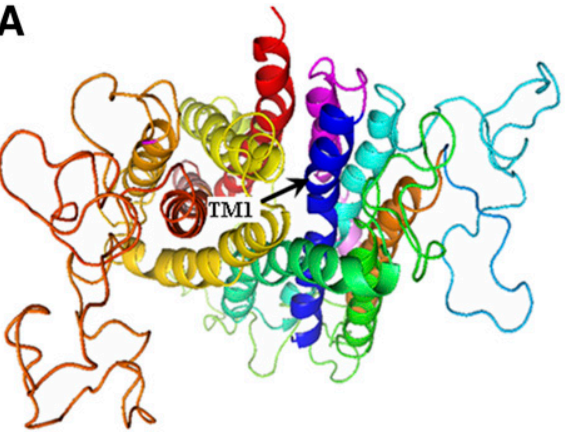

B

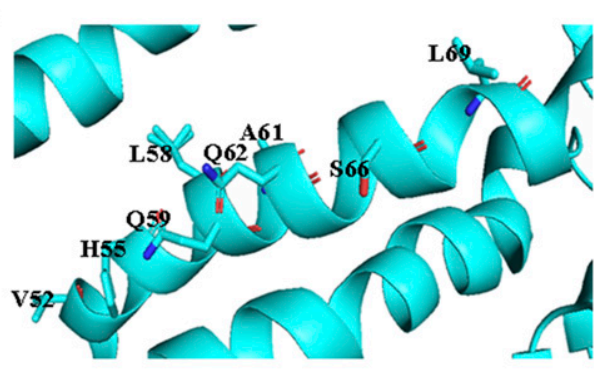

Fig. 8. Putative three-dimensional structural model of OATP2B1. (A) Viewed from the extracellular side of OATP2B1. Location of transmembrane domain 1 is indicated with an arrow. (B) Possible locations of the identified critical amino acid residues along TM1 of OATP2B1. The E. coli glycerol-3-phosphate transporter (Protein Data Bank: 1pw4) was used as the template for homology modeling of OATP2B1. The structure of OATP2B1 was modeled with the webbased protein structure prediction service SWISS-MODEL (https://www.swissmodel. expasy.org/). 
0.0002 was observed in OATP2B1. Based on our current study, such a mutation may affect uptake function of the transporter. Coordination analysis of results from biochemical studies with missense mutations reported in the National Center for Biotechnology Information database may help us better understand the therapeutic significance of OATP2B1 transmembrane domains.

\section{Authorship Contributions}

Participated in research design: Hong.

Conducted experiments: Fang, Huang, Chen, Xu, Xiang.

Performed data analysis: Huang, Hong.

Wrote or contributed to the writing of the manuscript: Hong.

\section{References}

Bian J, Jin M, Yue M, Wang M, Zhang H, and Gui C (2016) Tryptophan residue located at the middle of putative transmembrane domain 11 is critical for the function of organic anion transporting polypeptide 2B1. Mol Pharm 13:3553-3563.

Biasini M, Bienert S, Waterhouse A, Arnold K, Studer G, Schmidt T, Kiefer F, Gallo Cassarino T, Bertoni M, Bordoli L, et al. (2014) SWISS-MODEL: modelling protein tertiary and quaternary structure using evolutionary information. Nucleic Acids Res 42:W252-W258.

Chan T, Zheng J, Zhu L, Grewal T, Murray M, and Zhou F (2015) Putative transmembrane domain 6 of the human organic anion transporting polypeptide 1A2 (OATP1A2) influences transporter substrate binding, protein trafficking, and quality control. Mol Pharm 12:111-119.

Ciechanover A (2005) Proteolysis: from the lysosome to ubiquitin and the proteasome. Nat Rev Mol Cell Biol 6:79-87.

DeGorter MK, Ho RH, Leake BF, Tirona RG, and Kim RB (2012) Interaction of three regiospecific amino acid residues is required for OATP1B1 gain of OATP1B3 substrate specificity. Mol Pharm 9:986-995.

Glaeser H, Mandery K, Sticht H, Fromm MF, and König J (2010) Relevance of conserved lysine and arginine residues in transmembrane helices for the transport activity of organic anion transporting polypeptide 1B3. $\mathrm{Br} J$ Pharmacol 159 $698-708$.

Grube M, Köck K, Karner S, Reuther S, Ritter CA, Jedlitschky G, and Kroemer HK (2006) Modification of OATP2B1-mediated transport by steroid hormones. Mol Pharmacol 70:1735-1741.

Gui C and Hagenbuch B (2008) Amino acid residues in transmembrane domain 10 of organic anion transporting polypeptide 1B3 are critical for cholecystokinin octapeptide transport. Biochemistry 47:9090-9097.

Gui C and Hagenbuch B (2009) Role of transmembrane domain 10 for the function of organic anion transporting polypeptide 1B1. Protein Sci 18:2298-2306.

Hagenbuch B and Gui C (2008) Xenobiotic transporters of the human organic anion transporting polypeptides (OATP) family. Xenobiotica 38:778-801.

Hagenbuch B and Meier PJ (2003) The superfamily of organic anion transporting polypeptides. Biochim Biophys Acta 1609:1-18.

Hagenbuch B and Meier PJ (2004) Organic anion transporting polypeptides of the OATP/SLC21 family: phylogenetic classification as OATP/SLCO superfamily, new nomenclature and molecular/functional properties. Pflugers Arch 447:653-665.

Hong W, Wu Z, Fang Z, Huang J, Huang H, and Hong M (2015) Amino acid residues in the putative transmembrane domain 11 of human organic anion transporting polypeptide 1B1 dictate transporter substrate binding, stability, and trafficking. Mol Pharm 12:4270-4276.

Huang J, Li N, Hong W, Zhan K, Yu X, Huang H, and Hong M (2013) Conserved tryptophan residues within putative transmembrane domain 6 affect transport function of organic anion transporting polypeptide 1B1. Mol Pharmacol 84: $521-527$.

Kis O, Zastre JA, Ramaswamy M, and Bendayan R (2010) $\mathrm{pH}$ dependence of organic anion-transporting polypeptide 2B1 in Caco-2 cells: potential role in antiretroviral drug oral bioavailability and drug-drug interactions. J Pharmacol Exp Ther 334: 1009-1022.

Knauer MJ, Girdwood AJ, Kim RB, and Tirona RG (2013) Transport function and transcriptional regulation of a liver-enriched human organic anion transporting polypeptide 2B1 transcriptional start site variant. Mol Pharmacol 83:1218-1228.

Knauer MJ, Urquhart BL, Meyer zu Schwabedissen HE, Schwarz UI, Lemke CJ, Leake BF, Kim RB, and Tirona RG (2010) Human skeletal muscle drug transporters determine local exposure and toxicity of statins. Circ Res 106: 297-306.

Kobayashi D, Nozawa T, Imai K, Nezu J, Tsuji A, and Tamai I (2003) Involvement of human organic anion transporting polypeptide OATP-B (SLC21A9) in $\mathrm{pH}$-dependent transport across intestinal apical membrane. J Pharmacol Exp Ther 306:703-708

König J, Seithel A, Gradhand U, and Fromm MF (2006) Pharmacogenomics of human OATP transporters. Naunyn Schmiedebergs Arch Pharmacol 372:432-443.

Kullak-Ublick GA, Ismair MG, Stieger B, Landmann L, Huber R, Pizzagalli F, Fattinger K, Meier PJ, and Hagenbuch B (2001) Organic anion-transporting polypeptide B (OATP-B) and its functional comparison with three other OATPs of human liver. Gastroenterology 120:525-533.

Li N, Hong W, Huang H, Lu H, Lin G, and Hong M (2012) Identification of amino acids essential for estrone-3-sulfate transport within transmembrane domain 2 of organic anion transporting polypeptide 1B1. PLoS One 7:e36647.

Meier-Abt F, Mokrab Y, and Mizuguchi K (2005) Organic anion transporting polypeptides of the OATP/SLCO superfamily: identification of new members in nonmammalian species, comparative modeling and a potential transport mode. $J$ Membr Biol 208:213-227.

Miyagawa M, Maeda K, Aoyama A, and Sugiyama Y (2009) The eighth and ninth transmembrane domains in organic anion transporting polypeptide 1B1 affect the transport kinetics of estrone-3-sulfate and estradiol-17beta-D-glucuronide. $J$ Pharmacol Exp Ther 329:551-557.

Nakanishi T and Tamai I (2012) Genetic polymorphisms of OATP transporters and their impact on intestinal absorption and hepatic disposition of drugs. Drug Metab Pharmacokinet 27:106-121.

Niessen J, Jedlitschky G, Grube M, Bien S, Schwertz H, Ohtsuki S, Kawakami H, Kamiie J, Oswald S, Starke K, et al. (2009) Human platelets express organic aniontransporting peptide 2B1, an uptake transporter for atorvastatin. Drug Metab Dispos 37:1129-1137.

Nozawa T, Imai K, Nezu J, Tsuji A, and Tamai I (2004) Functional characterization of $\mathrm{pH}$-sensitive organic anion transporting polypeptide OATP-B in human. $J$ Pharmacol Exp Ther 308:438-445.

Ohnishi S, Hays A, and Hagenbuch B (2014) Cysteine scanning mutagenesis of transmembrane domain 10 in organic anion transporting polypeptide 1B1. Biochemistry 53:2261-2270.

Poirier A, Funk C, Lavé T, and Noé J (2007) New strategies to address drug-drug interactions involving OATPs. Curr Opin Drug Discov Devel 10:74-83.

Sai Y, Kaneko Y, Ito S, Mitsuoka K, Kato Y, Tamai I, Artursson P, and Tsuji A (2006) Predominant contribution of organic anion transporting polypeptide OATP-B (OATP2B1) to apical uptake of estrone-3-sulfate by human intestinal Caco-2 cells. Drug Metab Dispos 34:1423-1431.

Shirasaka Y, Mori T, Shichiri M, Nakanishi T, and Tamai I (2012) Functional pleiotropy of organic anion transporting polypeptide OATP2B1 due to multiple binding sites. Drug Metab Pharmacokinet 27:360-364.

Shitara Y, Sato H, and Sugiyama Y (2005) Evaluation of drug-drug interaction in the hepatobiliary and renal transport of drugs. Annu Rev Pharmacol Toxicol 45: $689-723$

Stieger B and Hagenbuch B (2014) Organic anion-transporting polypeptides. Curr Top Membr 73:205-232.

Tamai I (2012) Oral drug delivery utilizing intestinal OATP transporters. Adv Drug Deliv Rev 64:508-514.

Tamai I, Nezu J, Uchino H, Sai Y, Oku A, Shimane M, and Tsuji A (2000) Molecular identification and characterization of novel members of the human organic anion transporter (OATP) family. Biochem Biophys Res Commun 273:251-260.

Tamai I, Takanaga H, Maeda H, Yabuuchi H, Sai Y, Suzuki Y, and Tsuji A (1997) Intestinal brush-border membrane transport of monocarboxylic acids mediated by proton-coupled transport and anion antiport mechanisms. J Pharm Pharmacol 49: $108-112$

Tymoczko JL, Berg JM, and Stryer L (2015) Biochemistry: A Short Course, 3rd ed, W. H. Freeman, New York.

Vavricka SR, Van Montfoort J, Ha HR, Meier PJ, and Fattinger K (2002) Interactions of rifamycin SV and rifampicin with organic anion uptake systems of human liver. Hepatology 36:164-172.

Weaver YM and Hagenbuch B (2010) Several conserved positively charged amino acids in OATP1B1 are involved in binding or translocation of different substrates. J Membr Biol 236:279-290.

Address correspondence to: Mei Hong, College of Life Sciences, South China Agricultural University, Guangzhou, Gu 510642, China. E-mail: mh2788@scau.edu.cn 